\title{
Oculo-auriculo-vertebral spectrum: a review of the literature and genetic update
}

\author{
Ana Beleza-Meireles, ${ }^{1,2,3}$ Jill Clayton-Smith, ${ }^{3,4}$ Jorge M Saraiva, ${ }^{1}$ May Tassabehiji,4
}

\begin{abstract}
${ }^{1}$ Serviço de Genética, Departamento Pediátrico, Centro Hospitalar e Universitário de Coimbra, Coimbra, Portugal ${ }^{2}$ Center for Human Genetics, Cliniques Universitaires St Luc, Université Catholique de Louvain, Brussels, Belgium ${ }^{3}$ Faculty of Medical and Human Sciences, Manchester Centre for Genomic Medicine, Institute of Human Development, University of Manchester, Manchester, UK ${ }^{4}$ Central Manchester University Hospitals NHS Foundation Trust as part of Manchester Academic Health Science Centre (MAHSC), Manchester, UK
\end{abstract}

Correspondence to Dr May Tassabehji, Faculty of Medical and Human Sciences, Manchester Centre for Genomic Medicine, Institute of Human Development, University of Manchester, Manchester M13 OJH, UK: m.tassabehji@manchester.ac. uk

Received 28 May 2014 Revised 14 July 2014 Accepted 15 July 2014

\begin{abstract}
Oculo-auriculo-vertebral spectrum (OAVS, OMIM

$164210)$ is a developmental disorder primarily involving structures derived from the first and second pharyngeal arches during embryogenesis. The phenotype is clinically heterogeneous and is typically characterised by abnormal development of the ear, mandible anomalies and defects of the vertebral column. OAVS may occur as a multiple congenital abnormality, and associated findings include anomalies of the eye, brain, heart, kidneys and other organs and systems. Both genetic and environmental factors are thought to contribute to this craniofacial condition, however, the mechanisms are still poorly understood. Here, we present a review of the literature on OAVS, discussing what is known about the aetiology, candidate loci, possible mechanisms and the range of clinical features that characterise this condition. We also comment on some important aspects of recurrence risk counselling to aid clinical management.
\end{abstract}

\section{OVERVIEW OF OCULO-AURICULO-VERTEBRAL SPECTRUM}

Oculo-auriculo-vertebral spectrum (OAVS) is a disorder of craniofacial morphogenesis, ${ }^{1}{ }_{2}^{2}$ with a reported prevalence of up to $1 / 3500$ births. $^{3-6}$ It includes a group of malformations primarily involving structures derived from the first and second pharyngeal arches, in particular the ear, mouth and mandible. $^{7-28}$ OAVS is aetiologically and pathogenetically heterogeneous. As a result of this, many terms have been used to designate this complex spectrum of anomalies, such as hemifacial microsomia, first and second pharyngeal arch anomalies, facio-auriculo-vertebral syndrome and Goldenhar syndrome. The term OAVS has its origin from the designation 'oculoauriculovertebral dysplasia', suggested by Cohen et al (1989), ${ }^{2}$ who noted that there was a significant overlap between the different entities described above and suggested that they represent a phenotypic continuum.

Generally, the OAVS phenotype can range from mild to severe. Craniofacial abnormalities include asymmetric ear anomalies (preauricular tags and pits, ear dysplasia, anotia, microtia) with or without hearing loss (conductive and/or sensorineural); hemifacial microsomia resulting in facial asymmetry; orofacial clefts; ocular defects (epibulbar dermoids, microphthalmia, coloboma of upper eyelid); and vertebral abnormalities. Facial abnormalities are limited to one side in many, but not all cases. The majority of patients have some degree of facial asymmetry. ${ }^{13}$ When bilateral involvement is present, most of them demonstrate asymmetric involvement ${ }^{4} 18$ with more severe expression on one side. ${ }^{1}$ The right side appears to be more frequently involved. ${ }^{18}$ Other features such as cardiac, genitourinary and cerebral malformations have also been reported. ${ }^{7}$ Since not all patients with OAVS present with all the common features, microtia is accepted as the minimum criterion for diagnosis, but microtia, facial asymmetry and epibulbar dermoids/lipodermoids are considered 'typical' phenotypes.

OAVS usually occurs sporadically. However, segregation analysis has suggested genetic transmission in some cases. ${ }^{29}$ Reports of familial cases following autosomal dominant ${ }^{30-33}$ or autosomal recessive inheritance, ${ }^{43435}$ as well as evidence for genetic linkage in two families, ${ }^{36}{ }^{37}$ and the presence of OAVS features in patients with various chromosomal aberrations and genomic imbalances, ${ }^{35-67}$ all suggest that OAVS has a genetic basis in some cases. Environmental causes have also been suggested, such as maternal diabetes during pregnancy, gestational or pre-existing vasoactive drugs, smoking and twinning, indicating that a multifactorial aetiology (environmental and genetic) also contributes to some cases of OAVS. ${ }^{68-85}$

\section{The OAVS clinical phenotype}

The spectrum of phenotypic features in OAVS is variable, ranging from subtle facial asymmetry with a small skin tag in front of an otherwise normalappearing ear, to a complex phenotype comprising multiple congenital abnormalities (see figure 1 and table 1 for summary of phenotypes and their prevalence in 158 published cases $\left.{ }^{7-10}\right)$. Due to the variable expressivity, there is no consensus regarding the minimum diagnostic criteria for OAVS. Tasse et $\mathrm{al}^{7}$ suggested either isolated microtia or hemifacial microsomia together with mild ear malformations, such as preauricular tags and hillocks, (suggested to be variants of microtia) as minimal diagnostic criteria. From our clinical experience of the condition and evidence from the literature, we suggest that the presence of isolated hemifacial microsomia associated with a family history of OAVS should also be considered to be diagnostic.

Nearly all patients with OAVS have some degree of hemifacial microsomia, resulting from maxillary and/or mandibular hypoplasia (see summary of phenotypes in table 1). External ear abnormalities are also very common and include microtia, anotia, aural atresia, preauricular tags or hillocks and preauricular pits. Most affected individuals have some degree of hearing loss, therefore, all patients with OAVS need to have an audiological evaluation. Facial clefts, or cleft lip and/or palate may be observed, but these are not common features; but macrostomia is frequently seen. The craniofacial involvement is most commonly unilateral; but it 

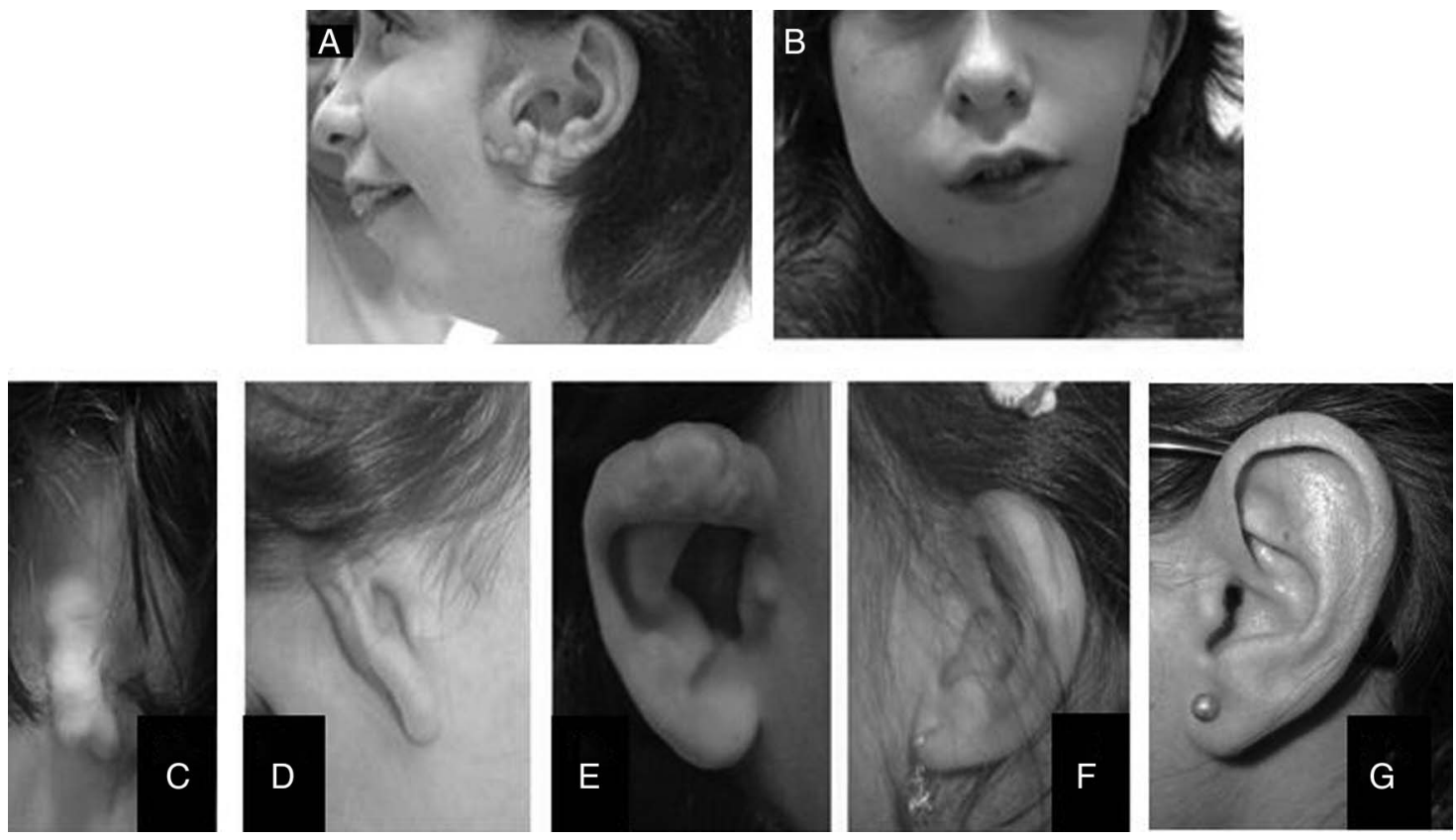

Figure 1 Characteristic craniofacial features of oculo-auriculo-vertebral spectrum (OAVS). (A-B) Two girls with OAVS, showing hemifacial microsomia and external ear defects. The first girl presents right hemifacial microsomia and right microtia, with agenesis of the external auditory meatus; she has no facial palsy, and her visual acuity is normal. The second girl has a left hemifacial microsomia and a displastic right ear with preauricular tags and displaced tragus; she has no facial palsy, and her visual acuity is normal. Neither had any additional malformations. (C-F) Different severities of microtia. The auricle is formed by a series of auricular hillocks that surround the 1st pharyngeal groove during the 6th week of gestation. At the initial stages, the auricle is formed at the base of the neck, but as the mandible develops, the auricles migrate to their normal anatomical position. Microtia occurs when the structures that form the auricle fail to develop normally. Several authors define four types of microtia according to the level of severity (type I, when the external ear is small, the auricle retains most of its normal structure, including the external auditory; type II, when the external ear is moderately anomalous, including hook-shaped, S-shaped or question mark-shaped auricle; type III, when the external is a rudimentary soft tissue structure with no cartilage and the auricle does not have a normal appearance; and type IV, characterised by anotia and absence of all external ear structures). Most cases of microtia may be difficult to define as the different forms of microtia-anotia follow a spectrum as opposed to tight groups as depicted in these photos. $(G)$ normal ear.

can also be bilateral, usually asymmetrical. Facial palsy, asymmetric palatal elevation, impairment of extraocular movements and trigeminal anaesthesia have been described in OAVS.

A variety of ocular abnormalities may also be observed, although less frequently than the name of the condition would suggest. Epibulbar dermoids is the most common ocular findings. Microphthalmia or other severe eye malformations may also be present but are rare. Spine deformities, impaired mobility of the spine, restricted neck movements and torticollis should raise the suspicion of vertebral anomalies in patients with OAVS. Moreover, congenital heart defects (tetralogy of Fallot, septal defects, transposition of the great vessels, aortic arch anomalies, situs inversus, dextrocardia) are not uncommon in patients with OAVS, ${ }^{7-10}$ and screening for structural cardiac anomalies should be part of the clinical evaluation of these patients.

Limb (particularly radial anomalies), renal (unilateral kidney agenesis, double ureter, renal ectopia, hydronephrosis, hydroureter) and central nervous system (developmental delay, microcephaly, encephalocele, hydrocephaly, hypoplasia of the corpus callosum, Arnold-Chiari malformation, holoprosencephaly) anomalies have also been observed in patients with OAVS. ${ }^{1} 27-25$

\section{The aetiology of OAVS}

Although knowledge of the genetic basis of human disease and its effect on embryonic development has greatly expanded in recent years, the causes of OAVS are still largely unknown, and the involvement of both genetic and environmental factors have been suggested. ${ }^{19}$ OAVS involves primarily the derivatives of the first and second pharyngeal arches, so it has been proposed that the aetiology and mechanisms of OAVS are related to the development of these structures. The pharyngeal arches, which start to develop in the 4th week of embryonic development, are composed of mesenchymal cells and give rise to various facial structures, including skeletal, muscular and neural elements, through a complex but poorly characterised signalling network. ${ }^{26-28}$ The morphogenesis of the pharyngeal arch derivatives depends on continuous and reciprocal tissue-tissue interactions. One of the key features of craniofacial development is the formation of cranial neural crest cells, which migrate ventrolaterally as they populate the craniofacial regions. Disturbances in the specification, migration, proliferation, survival and ultimate fate determination of the cranial neural crest cells have been proposed as a possible mechanism for OAVS. ${ }^{26-28}$ The phenotypic characteristics of OAVS and severity of the defects probably depend on how the expression and activation of certain developmental genes and proteins have been disrupted during facial development.

Microtia is a common phenotype in OAVS, which may arise as a consequence of neural crest cell defects and/or vascular disruption. This clinical feature can occur as an isolated defect or in association with other anomalies, such as Treacher Collins (TCOF1-3 mutations) and Branchio-oto-renal (EYA1, SIX1, SIX5) syndromes. ${ }^{86} 87$ Although the role of these genes in external ear development is not clearly defined, it does show that single gene defects can cause this phenotype by interfering in 
Table 1 Comparison of the prevalence of phenotypes from 158 published cases (7-10). (A) Summary of phenotype prevalence rates from 19 reports published between 1983 and 1996 as described by Cousley \& Calvert, $1997,{ }^{8}$ and adapted by Heike \& Hing, $2009 .{ }^{9}$

(B) Clinical evaluation of 53 patients $^{7}$; (C) Analysis of a cohort of 86 patients $^{10}$

\begin{tabular}{|c|c|c|c|}
\hline Principal anomalies & Study A $(\%)^{89}$ & Study B $(\%)^{7}$ & Study C $(\%)^{10}$ \\
\hline \multicolumn{4}{|l|}{ Head and face* } \\
\hline Hemifacial microsomiat & - & 83 & 84 \\
\hline Macrocephaly & - & - & 5 \\
\hline Microcephaly & - & 8 & - \\
\hline Cleft lip/palate & $15-22$ & & 18 \\
\hline $\begin{array}{l}\text { Macrostomia/facial } \\
\text { cleft }\end{array}$ & 1762 & & 13 \\
\hline Facial nerve palsy & $10-45$ & & - \\
\hline \multicolumn{4}{|l|}{ Ear } \\
\hline Anotia or microtia† & $66-99$ & $100 \ddagger$ & 70 \\
\hline Preauricular tags $\dagger$ & $34-61$ & & 67 \\
\hline Preauricular sinus/pit & $6-9$ & & 7 \\
\hline Hearing loss & $50-66 \S$ & 85 & 68 \\
\hline \multicolumn{4}{|l|}{ Ocular } \\
\hline Epibulbar dermoids & $4-35$ & 22 & 31 \\
\hline $\begin{array}{l}\text { Coloboma of the } \\
\text { upper eyelid }\end{array}$ & $12-25$ & 8 & 11 \\
\hline Microphthalmia & - & 10 & 12 \\
\hline Orbital dystopia & $15-43$ & - & - \\
\hline $\begin{array}{l}\text { Lacrimal duct atresia/ } \\
\text { stenosis }\end{array}$ & $11-14$ & - & - \\
\hline $\begin{array}{l}\text { Velopharyngeal } \\
\text { insufficiency }\end{array}$ & $35-55$ & - & - \\
\hline Vertebral anomalies & $16-609$ & 53 & 35 \\
\hline Congenital heart defects & $4-33$ & 15 & 27 \\
\hline Anomalies of extremities & $3-21$ & 12 & - \\
\hline Developmental delay & - & $9^{* *}$ & 14 \\
\hline $\begin{array}{l}\text { Central nervous system } \\
\text { anomalies }\end{array}$ & $5-18$ & $17+t$ & $2+t$ \\
\hline Urogenital anomalies & $4-15$ & 18 & $7 \ddagger \ddagger$ \\
\hline Pulmonary anomalies & $1-15$ & - & - \\
\hline $\begin{array}{l}\text { Gastrointestinal } \\
\text { anomalies }\end{array}$ & $2-12$ & - & - \\
\hline 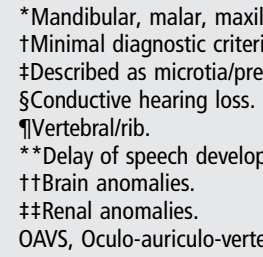 & $\begin{array}{l}\text { ary, or facial muscul } \\
\text { for OAVS. } \\
\text { uricular tag. } \\
\text { nent. }\end{array}$ & r hypoplasia. & \\
\hline
\end{tabular}

the genetic pathways necessary for normal development of mesenchymal tissues during fetal development.

Most OAVS cases are sporadic with no relevant family history. However, a genetic predisposition has been proposed based on growing evidence from the literature. ${ }^{29-34}$ Segregation analysis performed in 311 members of the families of 74 probands with OAVS provided evidence for an autosomal dominant mode of inheritance with reduced penetrance. ${ }^{29}$ Interestingly, a positive family history has been documented in only $2 \%$ of patients with OAVS, ${ }^{30-34}$ however, from our clinical experience of the condition, we believe that this number is an underestimate, and it is noteworthy that a recent report by Rooryck et al ${ }^{10}$ identified $12 \%$ of familial cases in a cohort of 95 patients. An additional clinical study by Rollnick et $a l^{19}$ has presented pedigree data on 97 cases, 44 of whom had a family history of the same or similar anomaly,
Table 2 Published chromosomal anomalies in patients with phenotypic characteristics of OAVS

\begin{tabular}{ll}
\hline Chromosomal abnormality & Reference \\
\hline Deletion in 1p22.2-p31.1 & 35 \\
Deletion 5q13.2 & 37 \\
Deletion 5p15 (5p15.33-pter) & 39404142 \\
Duplication 10p14-p15 & 43 \\
Deletion in 12p13.33 & 4445 \\
Duplication 14q23.1 & 4647 \\
Deletion 14q31.1q31.3 & 48 \\
Deletion 15q24.1 & 49 \\
Trisomy 18 & 50 \\
Deletion 22qter & 51 \\
Deletions 22q11.2 & 525354 \\
Trisomy 22 & 55 \\
Duplication 22q11.1-q11.21 & 5657 \\
Partial 22 trisomy of the 22q11 region & 58 \\
X chromosome aneuploidies & 596061 \\
Translocation t(9;18)(p23;q12.2) & 62 \\
Inversion inv9(p11;q13) & 63 \\
Inversion inv14(p11.2;q22.3) & 64 \\
Mosaicism of trisomy 7 & 65 \\
Mosaicism of trisomy 9 & 6667 \\
Mosaicism of trisomy 22 & 66 \\
\hline
\end{tabular}

even if it was only minor OAVS manifestations (minor external ear anomalies or preauricular tag, which might have been removed, being the most common). The authors have reported that firstdegree relatives were most often affected (35/433, 8\%). Of 176 sibs cited, $11(6 \%)$ were considered affected. In view of the family segregation of these anomalies, the authors suggest a multifactorial determination. Furthermore, the authors observe a broad phenotypic spectrum within families, an aspect that has also been reported by others, ${ }^{17}{ }^{18}$ and have proposed that familial inheritance is more frequent than originally reported. ${ }^{19}$ The evidence, so far, does not suggest that the familial OAVS phenotype is different from the sporadic OAVS cases.

Additional evidence for a genetic basis for OAVS comes from the identification of chromosomal anomalies in patients with phenotypic characteristics of this spectrum (summarised in table 2). Some of these abnormalities are recurrent. The $5 \mathrm{p} 15$ deletion has been observed in several patients with OAVS features; ${ }^{39-41}$ the region was further narrowed down to $5 \mathrm{p} 15.33$-pter by Ala-Mello, et al ${ }^{42}$ Deletions of the $12 \mathrm{p} 13.33$ region, involving the WNT5B gene, were observed in some but not all patients with OAVS features. ${ }^{44} 45$ Partially overlapping microduplications on $14 \mathrm{q} 23.1$ were identified in two families with autosomal dominant OAVS; ${ }^{46} 47$ one of these families included two first-degree relatives with clinical features of OAVS and Branchio-oto-renal syndrome; hence, the region $14 q 23.1$ might harbour candidate genes for OAVS and additional first and second pharyngeal arch developmental disorders. Anomalies in $22 \mathrm{q}$ have been frequently documented in patients with OAVS, particularly the 22qter deletion, ${ }^{51} 22 \mathrm{q} 11.2$ deletions, ${ }^{52-54}$ the $22 \mathrm{q} 11.1-\mathrm{q} 11.21$ (Cat-eye) region, ${ }^{56} 57$ and a partial 22 trisomy $(47, \mathrm{XX},+\operatorname{der}(22) \mathrm{t}(11 ; 22)(\mathrm{q} 23 ; \mathrm{q} 11))$, which duplicates the $22 \mathrm{q} 11$ region. ${ }^{66}$ Chromosomal mosaicism for trisomy $22^{66}$ has also been described, making this region a good candidate for some cases of OAVS.

Other candidate single gene loci for OAVS have also been suggested. Kelberman et al, ${ }^{36}$ reported linkage to a region of approximately $10.7 \mathrm{cM}$ on chromosome 14q32 (D14S987 and 
Table 3 aCGH CNV loci reported in patients with OAVS

\begin{tabular}{|c|c|c|c|c|c|c|}
\hline $\begin{array}{l}\text { Dosage anomaly } \\
\text { (reference) }\end{array}$ & Sequence coordinates & Phenotype & Genes in interval & $\begin{array}{l}\text { Minimum } \\
\text { size }(k b)\end{array}$ & Inheritance & Array CGH platform \\
\hline Del $1 p 22.2-p 31.1^{35}$ & $\begin{array}{l}\text { Distance from pter Mb band } \\
74-89.5\end{array}$ & $\begin{array}{l}\text { Craniofacial dysmorphism: facial asymmetry, R epibulbar } \\
\text { dermoid cyst, downslanting palpebral fissures, long nose, } \\
\text { small mouth, high arched palate, and teeth abnormalities; } \\
\text { narrow ears. } \\
\text { Additional anomalies: Psychomotor delay; short stature; } \\
\text { hypermetropia; conductive hearing loss; short neck, } \\
\text { scoliosis; cryptorchidism. }\end{array}$ & $\begin{array}{l}\text { FPGT, CRYZ, ACADM, RABGGTB, MSH4, ST6GALNAC3, } \\
\text { ST6GALNAC6, PIGK, AK5, FUBP1, PTGFR, IFI44, } \\
\text { LPHN2, PRKACB, DNASE2B, GNG5, CTBS, SSX2IP, } \\
\text { EDG7, MCOLN2, MCOLN3, BCL10, DDAHI, CYR61, } \\
\text { COL24A1, CLCA1/ CLCA2/ CLCA3, SH3GLB1, SEP15, } \\
\text { HS2ST1, LMO4, PKN2, GTF2B,KAT3, GBP1/ GBP2, } \\
\text { GBP3 }\end{array}$ & 15600 & $\mathrm{n} / \mathrm{a}$ & $\begin{array}{l}\text { High-resolution karyotype } \\
\text { and FISH + Genosensor } \\
\text { Array } 300 \text { aCGH }\end{array}$ \\
\hline Del $2 p 11.2^{10}$ & $87552703-89275144$ & $\begin{array}{l}\text { Craniofacial dysmorphism: L mandibular hypoplasia, cleft } \\
\text { palate, micrognathia; L microtia, R preauricular tags, L } \\
\text { pits. } \\
\text { Additional anomalies: not described }\end{array}$ & $\begin{array}{l}\text { RGPD2, PLGLB1, PLGLB2, KRCC1, SMYD1, FABP1, } \\
\text { THNSL2, C2orf51, EIF2AK3, RPIA }\end{array}$ & 1722 & $F(P)$ & $105 \mathrm{~K}$ Agilent Technologies \\
\hline Del $2 q 11^{10}$ & $99119770-99167676$ & $\begin{array}{l}\text { Craniofacial dysmorphism: maxillary and facial } \\
\text { musculature hypoplasia, mandibular hypoplasia, L } \\
\text { preauricular tags, L epibulbar dermoids. } \\
\text { Additional anomalies: not described }\end{array}$ & TSGA10, C2orf15, LIPT1, MITD1, MRPL30 & 48 & $\mathrm{~F}(\mathrm{M})$ & $44 \mathrm{~K}$ Agilent Technologies \\
\hline $\begin{array}{l}\text { Dup } \\
4 q 35.113 q 13.1^{10}\end{array}$ & $\begin{array}{l}186295254-86317114 \\
79675676-81318868\end{array}$ & $\begin{array}{l}\text { Craniofacial dysmorphism: L mandibular hypoplasia, B } \\
\text { preauricular tags, R pit, R epibulbar dermoids. } \\
\text { Additional anomalies: IUGR; agenesis of the 12th rib pair. }\end{array}$ & SLC25A4, KIAA1 430, SPRY2 & 221643 & $\mathrm{n} / \mathrm{a}$ & $105 \mathrm{~K}$ Agilent Technologies \\
\hline $\begin{array}{l}\text { Del } 5 \\
(p \operatorname{pter} \rightarrow \mathrm{p} 15.33)^{42}\end{array}$ & $\begin{array}{l}(45, X X, \operatorname{inv}(2)(q 32 q 37) m a t, \\
\operatorname{dic}(5 ; 21)(p 15.3 ; q 22.3) d n)\end{array}$ & $\begin{array}{l}\text { Craniofacial dysmorphism: upslanting palpebral fissures, } \\
\text { hypertelorism, epicanthi, epibulbar dermoids, broad nose; } \\
\text { 'asymmetric macrostomia' with } L \text { hemifacial microsomia } \\
\text { and cleft-like extension of the } L \text { corner of mouth; } L \text { cleft of } \\
\text { upper lip, submucous cleft palate and two preauricular } \\
\text { tags on } L \text {; normal ears. } \\
\text { Additional anomalies: language delay; IUGR, short stature; } \\
\text { recurrent middle ear infections; hypernasal voice; severe } \\
\text { myelodysplasia; history of neonatal trombocytopenia. }\end{array}$ & $\begin{array}{l}\text { PLEKHG4B, CCDC127, SDHA, PDCD6, AHRR, C5orf55, } \\
\text { EXOC3, SLC9A3, CTD-2228K2.7, CEP72, TPPP, } \\
\text { ZDHHC11, BRD9, TRIP13, NKD2, SLC12A7, SLC6A19, } \\
\text { SLC6A18, TERT, CLPTM1 L, SLC6A3, LPCAT1, MRPL36, } \\
\text { NDUFS6, IRX4, IRX2 (the smallest 5p deletion } \\
\text { associated to OAVS features) }\end{array}$ & 2000 & $S$ & $\begin{array}{l}44 \mathrm{~K} \text { and } 244 \mathrm{~K} \text { Agilent } \\
\text { Technologies }\end{array}$ \\
\hline Dup $8 q 11.23^{10}$ & $53635269-53719594$ & $\begin{array}{l}\text { Craniofacial dysmorphism: B preauricular tags, L epibulbar } \\
\text { dermoids. } \\
\text { Additional anomalies: not described }\end{array}$ & FAM150A, RB1CC1 & 84 & $\mathrm{~F}(\mathrm{M})$ & $105 \mathrm{~K}$ Agilent Technologies \\
\hline Dup $9 q 34.11^{10}$ & $130591031-130822006$ & $\begin{array}{l}\text { Craniofacial dysmorphism: high arched palate, B hearing } \\
\text { loss, B mandibular hypoplasia. } \\
\text { Additional anomalies: block vertebrae C3C4 }\end{array}$ & $\begin{array}{l}\text { TBC1D13, ENDOG, C9orf114, LRRC8A, PHYHD1, } \\
\text { CCBL1, DOLK, NUP188, SH3GLB2 }\end{array}$ & 230 & $F(P)$ & $105 \mathrm{~K}$ Agilent Technologies \\
\hline Dup 11q21 ${ }^{10}$ & 95725 560-95766 136 & $\begin{array}{l}\text { Craniofacial dysmorphism: } R \text { epibulbar dermoids, } \mathrm{L} \\
\text { mandibular hypoplasia, } \mathrm{L} \text { microtia with preauricular tags. } \\
\text { Additional anomalies: not described }\end{array}$ & CCD82, JRKL & 41 & $F(P)$ & $44 \mathrm{~K}$ Agilent Technologies \\
\hline Del $12 p 13.33^{10}$ & 0-2 309191 & $\begin{array}{l}\text { Craniofacial dysmorphism: } L \text { mandibular hypoplasia, wide } \\
\mathrm{L} \text { corner of the mouth, } \mathrm{L} \text { microtia, preauricular tags and } \\
\text { pits. } \\
\text { Additional anomalies: learning difficulties, IUGR, patent } \\
\text { formen ovale, short QT }\end{array}$ & $\begin{array}{l}\text { IQSEC3, SLC6A12, SLC6A13, JARID1A, CCDC77, } \\
\text { B4GALNT3, NINJ2, WNK1, HSN2, RAD52, ERC1, } \\
\text { FBXL14, WNT5B, ADIPOR2, CACNA2D4, LRTM2, } \\
\text { DCP1B, CACNA1C }\end{array}$ & 2309 & De novo & $44 \mathrm{~K}$ Agilent Technologies \\
\hline
\end{tabular}


Table 3 Continued

\begin{tabular}{|c|c|c|c|c|c|c|}
\hline $\begin{array}{l}\text { Dosage anomaly } \\
\text { (reference) }\end{array}$ & Sequence coordinates & Phenotype & Genes in interval & $\begin{array}{l}\text { Minimum } \\
\text { size }(k b)\end{array}$ & Inheritance & Array CGH platform \\
\hline $\begin{array}{l}\text { Del } 12 \\
(\mathrm{pter} \rightarrow \mathrm{p} 13.33)^{44}\end{array}$ & $\begin{array}{l}\text { between BAC RP11-275A6 } \\
\text { (73.9 Mb, 1p31.1) and RP5- } \\
\text { 908D6 (89.5 Mb, 1p22.2) }\end{array}$ & $\begin{array}{l}\text { Craniofacial dysmorphism: mild hypertelorism, broad nasal } \\
\text { tip, thin upper lip, wide } L \text { corner of the mouth, and } L \\
\text { hemifacial microsomia with } L \text { mandibular and condylar } \\
\text { hypoplasia; wide-spaced teeth; gingival hypertrophy; } \\
\text { bilateral dysplastic low-set ears with over-folded helix, } L \\
\text { microtia, } L \text { preauricular tag and pit. } \\
\text { Additional anomalies: delayed psychomotor development; } \\
\text { axial hypotonia and peripheral hypertonia; lumbar } \\
\text { kyphosis; patent foramen ovale; moderate } L \text { conductive } \\
\text { hearing loss. MRI showed } L \text { temporal arachnoid cyst with } \\
\text { widened subarachnoid spaces. History of IUGR and } \\
\text { polyhydramnios. }\end{array}$ & $\begin{array}{l}\text { IQSEC3, SLC6A12, SLC6A13, JARID1A, CCDC77, } \\
\text { BAGALNT3, NINJ2, WNK1, HSN2, RAD52, ERC1, } \\
\text { FBXL14, WNT5B*, ADIPOR2, CACNA2D4, LRTM2, } \\
\text { DCP1B, CACNA1C } \\
\text { (*highlighted by the authors as candidate gene.) }\end{array}$ & 2300 & $S$ & $44 \mathrm{~K}$ Agilent Technologies \\
\hline Dup $14 \mathrm{q} 23.1^{46}$ & $56278628-57623179$ & $\begin{array}{l}\text { Craniofacial dysmorphism: defects on aural, oral, } \\
\text { mandibular and vertebral development. } \\
\text { Additional anomalies: cardiac, pulmonary, renal, skeletal } \\
\text { and CNS anomalies. }\end{array}$ & $\begin{array}{l}\text { AK024921, BC041486,C14orf105, C14orf37, } \\
\text { LOC145407, EX9OC5, SEC10, MUDENG, NAT12, } \\
\text { OTX2*, SLC35F4 (*In common with }{ }^{47} \text { ) }\end{array}$ & 1034 & $A D$ & qChip-Post; qGenomics SL) \\
\hline $\begin{array}{l}\text { Dup } 14 \mathrm{q} 22.3- \\
\mathrm{q} 23.3^{47}\end{array}$ & $\begin{array}{l}14 \mathrm{q} 22.3 \text { at } \sim 55.774 \mathrm{Mb} \text { to } \\
14 \mathrm{q} 23.3 \text { at } \sim 67.571 \mathrm{Mb}\end{array}$ & $\begin{array}{l}\text { Craniofacial dysmorphism: short palpebral fissures an } \\
\text { lacrimal duct stenosis, bilateral facial clefts; short nose } \\
\text { with broad nasal bridge; micrognathia with severe } \\
\text { hypoplasia of mandible, high arched palate; multiple skin } \\
\text { tags and pits in preauricular and cheek regions; low-set } \\
\text { ears with abnormal antihelix and ear lobe L ear; } \\
\text { Eustachian tube dysfunction; adhesive otitis media. } \\
\text { Additional anomalies: IUGR; failure to thrive; short neck } \\
\text { with fusion of the pedicles and laminae on the L at C2-4; } \\
\text { hearing loss; torticollis; anterior ventricular septal defect; } \\
5 \mathrm{~cm} \text { meningocele and thin corpus callosum on MRI; deep } \\
\text { sacral dimple associated with conus tip at L3 with mild } \\
\text { thickening and fatty infiltration of the filum terminale; } \\
\text { small kidneys; hypoplastic genitalia. }\end{array}$ & $\begin{array}{l}\text { OTX2*, PSMA3, DAAM1, RTN1, SIX6*, SIX1*, SIX4, } \\
\text { MNAT1, PRKCH, HIF1A, KCNH5, PPP2R5E, SYNE2, } \\
\text { ESR2, ZNF46, HSPA2, SPTB, GPX2, FNTB, MAX, FUT8, } \\
\text { MPP5, PIGH, ARG2, RDH11, RDH12 (* SIX1 gene is } \\
\text { disease causing BOR and located at } 14 q 23.1)\end{array}$ & 11790 & $F(P) A D$ & $\begin{array}{l}244 \mathrm{~K} \text { and } 44 \mathrm{~K} \text { Agilent } \\
\text { Technologies }\end{array}$ \\
\hline $\begin{array}{l}\text { Del } 14 q 31.1- \\
q 31.3)^{48}\end{array}$ & $79617936-87566775$ & $\begin{array}{l}\text { Unilateral right-sided microtia, mild facial asymmetry; (CT) } \\
\text { scan unilateral aplasia of the middle cranial fossa floor on } \\
\text { the right, with a narrow external auditory duct; growth } \\
\text { retardation; no vertebral anomalies. Mother showed a } \\
\text { preauricular tag on R and hypothyroidism. }\end{array}$ & $\begin{array}{l}\text { DIO2, TSHR, GTF2AI, STON2, SELIL, FLRT2, GALC } \\
\text { (adjacent to GSC locus) }\end{array}$ & 7948 & $F(M)$ & $\begin{array}{l}\text { Karyotype and BAC FISH } \\
\text { CTD- } \\
\text { 2540C19 (AC010849) and } \\
\text { CTD-2357B9 (AQ062473) }\end{array}$ \\
\hline Del $14 q 32.2^{10}$ & $98250689-98253385$ & $\begin{array}{l}\text { Craniofacial dysmorphism: retrognathism; high arched } \\
\text { palate; } B \text { anotia, } B \text { mandibular hypoplasia }(\mathrm{R}>\mathrm{L}) \text {. } \\
\text { Additional anomalies: not described }\end{array}$ & C14orf177 & 2.7 & $\mathrm{~F}(\mathrm{M})$ & $105 \mathrm{~K}$ Agilent Technologies \\
\hline Del 15q24.1q24.249 & **74 419 546-75931476 & $\begin{array}{l}\text { Craniofacial dysmorphism: cleft of the soft palate, } \\
\text { hemifacial microsomia due to malar and mandibular } \\
\text { hypoplasia, R grade IV microtia. } \\
\text { Additional anomalies: moderate psychomotor delay and } \\
\text { learning difficulties, growth retardation; myopia and } \\
\text { strabismus; bilateral conductive hearing loss hypotonia; } \\
\text { acute dyspnoea episodes and recurrent respiratory } \\
\text { infections; gastrointestinal reflux; history of IUGR. }\end{array}$ & $\begin{array}{l}\text { STRA6, CCDC5, CYP11A1, UBL7, ARID3B, CLK3, } \\
\text { CPLX3, GOLGA6C, GOLGA6D, SIN3A, PTPN9, MAN2C, } \\
\text { CSK (according to the figure 2) }\end{array}$ & & $S$ & $60 \mathrm{~K}$ Agilent Technologies \\
\hline
\end{tabular}


Table 3 Continued

\begin{tabular}{|c|c|c|c|c|c|c|}
\hline $\begin{array}{l}\text { Dosage anomaly } \\
\text { (reference) }\end{array}$ & Sequence coordinates & Phenotype & Genes in interval & $\begin{array}{l}\text { Minimum } \\
\text { size }(k b)\end{array}$ & Inheritance & Array CGH platform \\
\hline Del $15 q 24^{49}$ & $\begin{array}{l}{ }^{*} * 70.708-73.856 \mathrm{Mb} \text { to } \\
{ }^{*} 74419546-75931476\end{array}$ & $\begin{array}{l}\text { Craniofacial dysmorphism: round, asymmetric face, smooth } \\
\text { philtrum cup-shaped protruding ears. } \\
\text { Additional anomalies: cognitive impairment, hypotonia; } \\
\text { recurrent ear infections, microphallus. }\end{array}$ & $\begin{array}{l}\text { STRA6, CYP11A1, SEMA7A, CSK, CPLX3, MPI, and } \\
\text { SIN3A (defined as the minimum critical region for the } \\
15 \text { q24 deletion syndrome) }\end{array}$ & 3148 & $S$ & $\begin{array}{l}\text { Chromosomal Microarray } \\
\text { Analysis (CMA Versions } 6 \\
\text { and } 7 \text { OLIGO) }\end{array}$ \\
\hline $\begin{array}{l}\text { Dup 18p11.23- } \\
\text { p11.31 }\end{array}$ & $6932021-8074805$ & $\begin{array}{l}\text { Craniofacial dysmorphism: mandibular hypoplasia; R grade } \\
\text { III microtia with preauricular tag. } \\
\text { Additional anomalies: not described }\end{array}$ & PTPRM, LRRC30, LAMA1 & 1142 & $F(P)$ & 244 K Agilent Technologies \\
\hline Dup 20p12.2 ${ }^{10}$ & 9971 936-10 124896 & $\begin{array}{l}\text { Craniofacial dysmorphism: } R \text { microphtalmia, R hemifacial } \\
\text { microsomia, R microtia with preauricular tag. } \\
\text { Additional anomalies: learning disabilities, hemivertebrae } \\
\text { T1, abnormal ribs, R renal agenesis. }\end{array}$ & ANKRD5 & 153 & $F(M)$ & $105 \mathrm{~K}$ Agilent Technologies \\
\hline $\begin{array}{l}\text { Del } 22 q 11.21- \\
q 11.22^{52}\end{array}$ & $\begin{array}{l}20128705-21246612 \\
20453484-20551970\end{array}$ & $\begin{array}{l}\text { Craniofacial dysmorphism: microcephaly, bilateral cleft lip } \\
\text { and palate, L macrostomia, multiple bilateral preauricular } \\
\text { tags, and small external auditory canals; } \\
\text { Additional anomalies: IUGR, developmental delay, multiple } \\
\text { ventricular septal defects. }\end{array}$ & $\begin{array}{l}\text { HIC2, LOC220686, UBE2L3, LOC150223, CCDC116, } \\
\text { SDF2L1, PPIL2, YPEL1, MAPK1, PPM1F, TOP3B, } \\
\text { VPREB1, LOC96610, SUHW2, SUHW1, PRAME }\end{array}$ & 1118 & $S$ & $\begin{array}{l}\text { High-resolution G-banding } \\
\text { ( } \sim 50 \text { band level) and } \\
\text { aCGH44 K array }\end{array}$ \\
\hline Del $22 q 11.2^{53}$ & $\begin{array}{l}\text { See N25 FISH probe } \\
\text { coordinates at the 22q11 } \\
\text { D22S75 locus (Vysis) }\end{array}$ & $\begin{array}{l}\text { Craniofacial dysmorphism: coloboma of upper } L \text { eyelid, } \\
\text { cleft palate, small mouth, asymmetric mandibular } \\
\text { hypoplasia, } L \text { hemifacial microsomia, bilateral preauricular } \\
\text { tags, microtia with aural atresia of } L \text { ear; absence of } \\
\text { external auditory meatus and dysgenesis of inner ear } \\
\text { system on } L \text {; hearing loss in } L \text { ear. } \\
\text { Additional anomalies: CHD (double outlet } R \text { ventricle with } \\
\text { valvular and infundibular pulmonary stenosis, subaortic } \\
\text { ventricular septal defect, and normally related great } \\
\text { arteries); hypocalcaemia secondary to hypoparathyroidism; } \\
\text { low T-lymphocyte count, normal serum immunoglobulins. }\end{array}$ & $\begin{array}{l}\text { Genes located inside the } 22 q 11.2 \text { critical region } \\
\text { including } T B X 1 \text { and UFD1L }\end{array}$ & $22 q 11.2$ & $S$ & FISH with N25 probe \\
\hline Del $22 q 11.2^{53}$ & $\begin{array}{l}\text { See N25 FISH probe } \\
\text { coordinates at the 22q11 } \\
\text { D22S75 locus (Vysis) }\end{array}$ & $\begin{array}{l}\text { Craniofacial dysmorphism: prominent forehead with a } \\
\text { receding hairline, mild facial asymmetry (smaller R cheek), } \\
\text { asymmetric crying facies, R facial droop; R ear microtia } \\
\text { with preauricular tag and atresia, low-set L ear with } \\
\text { overfolded, thick superior helix, R internal ear anomalies } \\
\text { (prominent vestibule, no lateral semicircular canal). } \\
\text { Additional anomalies: brain MRI showed open } \\
\text { fronto-temporal operculum with persistent cavum septum } \\
\text { pellucidum, mild widening of foramen of Magendie and } \\
\text { cisterna magna; feeding and respiratory difficulties, severe } \\
\text { gastro-oesophageal reflux; R-sided hearing loss; sacral } \\
\text { dimple, long fingers; CHD (aberrant R subclavian artery, } \\
\text { patent foramen ovalis, trivial tricuspid regurgitation). }\end{array}$ & $\begin{array}{l}\text { Genes located inside the } 22 q 11.2 \text { critical region } \\
\text { including TBX1 and UFD1L }\end{array}$ & $22 q 11.2$ & $S$ & FISH with N25 probe \\
\hline Del $22 q 11.2^{53}$ & $\begin{array}{l}\text { See N25 FISH probe } \\
\text { coordinates at the 22q11 } \\
\text { D22575 locus (Vysis) }\end{array}$ & $\begin{array}{l}\text { Craniofacial dysmorphism: hemifacial microsomia, } \\
\text { periorbital fullness, broad nasal root, bulbous nasal tip, } \\
\text { hypoplastic nares, submucous cleft palate, small mouth, L } \\
\text { microtia with aural atresia, small R ear with simple } \\
\text { protruding helix. }\end{array}$ & $\begin{array}{l}\text { Genes located inside the 22q11.2 critical region } \\
\text { including TBX1 and UFD1L }\end{array}$ & $22 q 11.2$ & De novo & FISH with N25 probe \\
\hline
\end{tabular}


Table 3 Continued

\begin{tabular}{|c|c|c|c|c|c|c|}
\hline $\begin{array}{l}\text { Dosage anomaly } \\
\text { (reference) }\end{array}$ & Sequence coordinates & Phenotype & Genes in interval & $\begin{array}{l}\text { Minimum } \\
\text { size }(k b)\end{array}$ & Inheritance & Array CGH platform \\
\hline & & $\begin{array}{l}\text { Additional anomalies: developmental delay; bilateral optic } \\
\text { nerve hypoplasia; R ear hearing loss; tapered fingers; } \\
\text { butterfly vertebra at T8 and T10 and sacral dimple; CHD } \\
\text { (small atrial septal defect ostium secundum type). } \\
\text { Dysphagia, feeding difficulties and laryngomalcia. }\end{array}$ & & & & \\
\hline Del $22 q 11.2^{54}$ & 19782 237-22 969069 & $\begin{array}{l}\text { Craniofacial dysmorphism: epibulbar dermoid, hemifacial } \\
\text { microsomia, bilateral ear tags } \\
\text { Additional anomalies: postnatal growth restriction (note, } \\
\text { maternal diabetes). }\end{array}$ & Not listed & 3200 & S & $\begin{array}{l}\text { Illumina HumanCytoSNP-12 } \\
\text { v1.0 BeadChip }\end{array}$ \\
\hline $\begin{array}{l}\text { Dup 22q11.1- } \\
\text { q11.21 }\end{array}$ & **17 381 569-18 618107 & $\begin{array}{l}\text { Craniofacial dysmorphism: frontal bossing, facial } \\
\text { asymmetry, flat nasal bridge, bilateral mandibular } \\
\text { hypoplasia with asymmetric retrognathia; asymetric ears } \\
\text { with simplified helices, preauricular ear tag on } L \text { and } \\
\text { larger ear tag associated with the tragal cartilage on the } \\
\mathrm{R} \text {, atretic } R \text { ear canal and associated sensorineural hearing } \\
\text { loss. } \\
\text { Additional anomalies: hypotonia; branchial cleft sinus, } \\
\text { R-sided muscular torticollis, esotropia, bilateral } \\
\text { clinodactyly, tapered fingers, L lower extremity shorter } \\
\text { than } R \text {, sacral dimple; sparse hair, }\end{array}$ & $\begin{array}{l}\text { CECR1, CECR2, CECR3, CECR5, CECR6, CECR7, GAB4, } \\
\text { AS1, IL17RA, ATP6V1E1, BCL2L13, BID, MIR3198-1, } \\
\text { MIR648, FL41941, PEX26, TUBA8, MICAL3, USP18, } \\
\text { G6T3P }\end{array}$ & 1500 & $S$ & $\begin{array}{l}\text { Combimatrix CMDX BAC } \\
\text { array+FISH (VYSIS probes } \\
\text { D22Z1, and TUPLE1) }\end{array}$ \\
\hline $\begin{array}{l}\text { Dup } \\
\text { 22q11.1q11.21 Del } \\
22 q 11.21 q 11.22^{57}\end{array}$ & $20000000-21 / 22000000$ & $\begin{array}{l}\text { Craniofacial dysmorphism: short stature and icrocephaly. } \\
\text { R-sided facial cleft; R-sided external auditory canal atresia } \\
\text { and bilateral conductive hearing loss; flattening of } \\
\text { zygoma; mandibular asymmetry; bilateral epicanthal folds; } \\
\text { myopia. } \\
\text { Additional anomalies: IQ of } 77 \text {; mild lumbar lordosis. }\end{array}$ & $\begin{array}{l}\text { 22q11.1q11.21 dup: IL17RA, CECR1, CECR2, } \\
\text { SLC25A18, ATP6V1E1, BID, MICAL3, PEX26, TUBA8, } \\
\text { and USP18. } \\
\text { 22q11.21q11.22 del: HIC2, RIMBP3B, RIMBP3C, } \\
\text { UBE2L3, SDF2L1, MIR130B, PPIL2, YPEL1, MAPK1, } \\
\text { TOP3B, VPREB1, PRAME, GGTLC2, and RTDR1. }\end{array}$ & 1100 & $\mathrm{n} / \mathrm{a}$ & $\begin{array}{l}\text { Nimblegen oligo12-plex array } \\
+ \text { FISH (RP11-829C4) }\end{array}$ \\
\hline $\begin{array}{l}\text { Dup } \\
\text { Yp-q11.221, Del } \\
\text { Yq11.222-q12 }\end{array}$ & $\begin{array}{l}2715688-18073734 \\
19492239-57432638\end{array}$ & $\begin{array}{l}\text { Craniofacial dysmorphism: L epibulbar dermoids, } \\
\text { micrognathia, R mandibular and maxillary hypoplasia, } \\
\text { macrostomia, cleft palate, B anotia. } \\
\text { Additional anomalies: IUGR; block cervical vertebrae. }\end{array}$ & Y chromosome (not all genes listed) & $\begin{array}{l}15000 \\
38000\end{array}$ & S & $105 \mathrm{~K}$ Agilent Technologies \\
\hline $\begin{array}{l}\text { Amplification } \\
\text { Xp22.33 }\end{array}$ & $3588423-3645922$ & $\begin{array}{l}\text { Craniofacial dysmorphism: } \mathrm{R} \text { epibulbar dermoids, } \mathrm{R} \\
\text { mandibular hypoplasia, } \mathrm{R} \text { preauricular tags. } \\
\text { Additional anomalies: not described }\end{array}$ & PRKX & 57 & $F(P)$ & $105 \mathrm{~K}$ Agilent Technologies \\
\hline Trisomy $X^{10}$ & & $\begin{array}{l}\text { Craniofacial dysmorphism: R hemifacial microsomia, } \\
\text { micrognathia, R microtia with preauricular tags and pits, } \\
\text { Additional anomalies: asymmetric legs and R breast } \\
\text { hypoplasia. }\end{array}$ & $\mathrm{X}$ chromosome (not all genes listed) & $\mathrm{x}$ & $S$ & $105 \mathrm{~K}$ Agilent Technologies \\
\hline
\end{tabular}

Genome builds: **GRCH37/hg19, all other genome loci are in NCBI36/hg18.

OAVS, Oculo-auriculo-vertebral spectrum; AD, autosomal dominant; CHD, congenital heart disease; Del, deletion; dup, duplication; F, familial; IUGR, intrauterine growth restriction; L, left; M, maternal; n/a, not available; None, no pathogenic changes; $P$, paternal; $R$, right; $S$, sporadic/de novo. 
D14S65) containing the GSC (GOOSECOID) gene in an OAVS family with apparent autosomal dominant inheritance. Additionally, Gimelli et $a{ }^{48}$ reported an interstitial $14 \mathrm{q} 31.1 \mathrm{q} 31.3$ deletion, a region adjacent to GSC, transmitted from a mother to her daughter, both with features of hemifacial microsomia. Although GSC could be regarded as a candidate gene, no mutation has been found in OAVS cases with normal karyotypes. However, GSC is now known to be mutated in a syndrome of short stature, auditory-canal atresia, mandibular hypoplasia and skeletal abnormalities (SAMS syndrome). ${ }^{88}$ This is a rare autosomal-recessive developmental disorder with a unique skeletal phenotype which, in our view, represents a different entity, clinically distinct from OAVS.

In another family with five OAVS patients, potential linkage was suggested at $15 \mathrm{q} 26.2$-q26.3. ${ }^{37}$ Moreover, 12 genomic rearrangements (4 deletions and 8 duplications) ranging from $2.7 \mathrm{~kb}$ to $2.3 \mathrm{Mb}$, were found by Rooryck et $a l,{ }^{10}$ by high-density oligonucleotide array comparative genomic hybridization $(\mathrm{aCGH})$ analysis (see table 3 ). These included a de novo 12 q13.33 deletion, which has previously been associated with OAVS $^{44}{ }^{45}$; a 2 q11 deletion, which was present in a proband and his affected maternal aunt; and trisomy $\mathrm{X}$ in a girl OAVS. Table 2 summarises the putative chromosomal loci for OAVS based on the abnormalities described in this review. In spite of growing evidence for high heritability, the molecular basis of OAVS is still elusive and, although Mendelian inheritance is more likely in familial cases of OAVS, sporadic cases may have polygenic or multifactorial causes.

There is no doubt that environmental factors, such as maternal diabetes during pregnancy, thalidomide, vasoactive drugs, smoking and multiple pregnancy may also play a part in causation of this condition. One of the most favoured hypotheses is that of vascular disruption of insufficiency in utero. ${ }^{68-71}$ In fact, disruption of embryonic blood flow during development, maternal vasoactive medication use, diabetes and twinning are predisposing factors to a number of structural congenital anomalies. $^{72} 73$ Moreover, a great number of case reports on concordant and discordant twins with OAVS suggest that there might be an association between reproductive abnormalities, twinning and OAVS. ${ }^{74-76}$ An excess of affected infants born after assisted reproductive techniques and frequent twinning among naturally conceived patients with OAVS has been reported. ${ }^{75}$ Furthermore, the high frequency of sporadic cases and the notable occurrence of discordant monozygotic twins defy simple Mendelian inheritance. ${ }^{7-83}$ It is worthwhile pointing out that some of these reports, in which an environmental exposure was considered causative, do not all mention whether or not the relatives were carefully evaluated in order to exclude the effect of genetic background.

Epigenetic mechanisms may be one of the ways to explain how genes and the environment interact to impair cranial neural crest cell development and cause this spectrum of craniofacial disorders. Both assisted reproduction and monozygotic twinning may predispose to epigenetic modification. Studies with model organisms reveal how the identity and patterning of vertebrate-specific portions of the skull are epigenetically regulated and how epigenetic dysregulation in cranial neural crest cells induces severe skull vault defects. ${ }^{84}$ Epigenetic factors have been suggested as a possible pathogenic mechanism leading to histone acetylation-dependent imbalance of allelic expression of the BAPX1 gene, a member of the NKX family of homeobox-containing proteins, which play a role in skeletal development and patterning of the middle ear, in five patients with OAVS. ${ }^{85}$
Heterogeneity encompassing genetic, epigenetic and nongenetic factors appears to be the most likely explanation for OAVS. A multifactorial and complex aetiology is a possible model to explain the occurrence of this birth defect. In support of this hypothesis, an increased recurrence risk was observed in families with an affected OAVS relative. ${ }^{17-20}$ The recurrence risk in first-degree relatives of affected individuals is estimated to be $2 \%-3 \%$ (as we have discussed, this is probably an underestimate). It has been proposed that the presence of rare and common genetic variation, of variable penetrance and effect, combined with environmental factors, may affect specific tissue interactions that occur between the cranial neural crest cells and the endoderm, mesoderm and ectoderm, and the way they connect during their migration in establishing the foundations of craniofacial morphogenesis, hence affecting the risk to OAVS.

\section{Differential diagnoses of OAVS}

Some patients with OAVS have clinical findings that overlap with other syndromes involving structures derived from the first and second pharyngeal arches. We highlight five syndromes with known causative genes in table 4: Treacher Collins syndrome, ${ }^{86}$ Townes-Brocks syndrome, ${ }^{89}$ CHARGE syndrome, ${ }^{90}$ Branchio-oto-renal spectrum disorders, ${ }^{91}$ and the phenotypic spectrum associated with mutations in EFTUD2..$^{92} 93$ Our view is that these conditions are distinctive and recognisable phenotypic entities. The presence of anal anomalies, for instance, points strongly towards Townes-Brock syndrome; the shape of the ear and semicircular canal abnormality in CHARGE syndrome are very characteristic, and individuals with EFTUD2 mutations have microcephaly and may also have associated oesophageal atresia. However, if features are sufficiently suggestive, molecular testing of the genes that cause these syndromes might be considered in some OAVS cases.

\section{RECOMMENDATIONS FOR GENETIC COUNSELLING}

The diagnosis of OAVS is frequently based only on clinical phenotyping, since no specific genetic tests are available and chromosome abnormalities have been identified in only a few cases. The lack of consensus regarding the minimum clinical diagnostic criteria for OAVS and the uncertainties regarding its aetiology cause difficulties in genetic counselling. An empiric sibling recurrence risk of $2 \%-3 \%$ is given in the case of a proband with OAVS, with normal chromosomes and no family history. However, one must be aware of occasional autosomal dominant or autosomal recessive inheritance observed in some cases. Before counselling these families, parents and siblings must be observed and the family history ascertainment must be thorough. The identification of subtle clinical manifestations of OAVS in relatives is important for recurrence risk counselling. A three-generation family history profile specifically enquiring about other relatives with facial asymmetry, ear tags, ear pits, hearing loss, vertebral malformations, eye abnormalities, cardiac or urogenital malformations and/or abnormalities of the extremities is essential.

If an individual with OAVS is found to have an inherited or de novo chromosome abnormality, genetic counselling for that condition is advised, although because of phenotypic heterogeneity, one cannot accurately predict the severity of OAVS should it occur in another child with the same chromosomal disorder. To illustrate this, we can take into account the phenotypic variability in genomic disorders such as those on 22q11 (Cat-eye syndrome, der(22) syndrome and velocardiofacial syndrome/ DiGeorge syndrome), where chromosomal rearrangements lead to abnormal gene dosage, resulting in phenotypes including intellectual disability and congenital malformations. ${ }^{94-99}$ 
Table 4 Differential diagnoses of OAVS

\begin{tabular}{|c|c|c|c|}
\hline Diagnosis & Main clinical features & Gene & Ref. \\
\hline $\begin{array}{l}\text { Treacher Collins syndrome } \\
\text { OMIM 154500, 613717, } 248390\end{array}$ & $\begin{array}{l}\text { Hypoplasia of the zygomatic bones and mandible } \\
\text { External ear abnormalities frequently associated with hearing impairment } \\
\text { Coloboma of the lower eyelid } \\
\text { Absence of the lower eyelashes } \\
\text { Preauricular hair displacement onto the cheeks } \\
\text { Craniofacial involvement is generally symmetrical }\end{array}$ & TCOF1 & 86 \\
\hline $\begin{array}{l}\text { Townes-Brocks syndrome } \\
\text { OMIM } 107480\end{array}$ & $\begin{array}{l}\text { Imperforate anus } \\
\text { Dysplastic ears (overfolded superior helices and preauricular tags) frequently } \\
\text { associated with sensorineural and/or conductive hearing impairment } \\
\text { Thumb malformations (triphalangeal thumbs, duplication of the thumb, preaxial } \\
\text { polydactyly or hypoplasia of the thumbs) } \\
\text { Renal impairment with or without structural abnormalities }\end{array}$ & SALL1 & 89 \\
\hline $\begin{array}{l}\text { CHARGE syndrome } \\
\text { OMIM } 214800\end{array}$ & $\begin{array}{l}\text { Coloboma of the iris, retina-choroid, and/or disc } \\
\text { Unilateral or bilateral choanal atresia or stenosis } \\
\text { Ear abnormalities (external ear malformation, ossicular malformations, Mondini defect } \\
\text { of the cochlea and/or absent/hypoplastic semicircular canals) } \\
\text { Cryptorchidism in males and hypogonadotrophic hypogonadism in both males and } \\
\text { females } \\
\text { Cardiovascular malformations } \\
\text { Orofacial clefts } \\
\text { Tracheoesophageal fistula } \\
\text { Cranial nerve dysfunction }\end{array}$ & CHD7 & 90 \\
\hline $\begin{array}{l}\text { Branchio-oto-renal spectrum disorders } \\
\text { (branchio-oto-renal and branchio-otic syndromes) } \\
\text { OMIM 113650, 610896, } 602588\end{array}$ & $\begin{array}{l}\text { Malformations of the outer, middle and inner ear } \\
\text { Conductive, sensorineural, or mixed hearing impairment } \\
\text { Branchial fistulae and cysts, } \\
\text { Renal malformations ranging from mild renal hypoplasia to bilateral renal agenesis. } \\
\text { Branchio-otic syndrome has the same features as branchio-oto-renal syndrome but } \\
\text { without renal involvement. }\end{array}$ & $\begin{array}{l}\text { EYA1, SIX5 } \\
\text { and SIX1 }\end{array}$ & 91 \\
\hline $\begin{array}{l}\text { Mandibulofacial dysostosis, Guion-Almeida-type } \\
\text { OMIM } 610536\end{array}$ & $\begin{array}{l}\text { Oto-facial abnormalities (acrofacial dysostosis) } \\
\text { Oesophageal atresia } \\
\text { Thumb anomalies } \\
\text { Intellectual disability } \\
\text { Zygomatic anomalies } \\
\text { - Microcephaly }\end{array}$ & EFTUD2 & 9293 \\
\hline
\end{tabular}

OAVS, oculo-auriculo-vertebral spectrum.

Therefore, although no specific genetic test is currently available, aCGH screening should be considered in patients with OAVS when recurrence risk counselling is sought, particularly in those with multiple anomalies and/or developmental delay.

Prenatal diagnosis of sporadic cases has been seldom reported and when achieved, has typically been in those with severe malformations, but with the advent of more detailed 3D scans, milder cases may be detectable (reviewed in Ref 9). Fetal ultrasound examination to detect microtia, preauricular tags and/or asymmetric mandibular hypoplasia is possible. However, since the genetic causes of OAVS have not been identified, prenatal diagnosis using genetic testing is not yet possible.

Understanding the molecular basis of these malformations will contribute to the identification of critical genes for craniofacial development and the identification of causative gene(s) will provide tools for diagnosis and genetic counselling of this complex disorder.

Acknowledgements We thank The Rosetrees Trust for the funding part of this study. We would also like to acknowledge the Department of Medical Genetics at the Paediatric Hospital in Coimbra, Portugal and the patients who consented to having their pictures published in this paper.

Contributors MT and AB-M researched and wrote the manuscript. JC-S edited and contributed to the manuscript.

Patient consent Obtained.

Competing interests None.

Ethics approval Ethical Review Board at Department of Medical Genetics, Coimbra University Hospital Centre (Centro Hospitalar e UniversitÃ jrio de Coimbra).

Provenance and peer review Not commissioned; externally peer reviewed.

\section{REFERENCES}

1 Hennekam RCM, Krantz ID, Allanson JE. Syndromes of the head and neck. 5thedn. Oxford, UK: Oxford University Press, 2010.

2 Gorlin RJ, Jue KL, Jacobsen U, Golschmidh E. Oculoauriculovertebral dysplasia. J Pediatr 1963;63:991-9.

3 Mastroiacovo P, Corchia C, Botto LD, Lanni R, Zampino G, Fusco D. Epidemiology and genetics of microtia-anotia: a registry based study on over one million births. J Med Genet 1995;32:453-7.

4 Grabb WC. The first and second branchial arch syndrome. Plast Reconstr Surg 1965;36:485-508.

5 Stoll C, Roth MP, Dott B, Bigel P. Discordance for skeletal and cardiac defect in monozygotic twins. Acta Genet Med Gemellol (Roma) 1984;33:501-4.

6 Poswillo D. Otomandibular deformity: pathogenesis as a guide to reconstruction. J Maxillofac Surg 1974;2:64-72.

7 Tasse $C$, Böhringer $S$, Fischer $S$, Lüdecke HJ, Albrecht B, Horn D, Janecke A, Kling R, König R, Lorenz B, Majewski F, Maeyens E, Meinecke P, Mitulla B, Mohr C, Preischl M, Umstadt H, Kohlhase J, Gillessen-Kaesbach G, Wieczorek D. Oculo-auriculo-vertebral spectrum (OAVS): clinical evaluation and severity scoring of 53 patients and proposal for a new classification. Eur J Med Genet 2005; 48:397-411.

8 Cousley RR, Calvert ML. Current concepts in the understanding and management of hemifacial microsomia. Br J Plast Surg 1997;50:536-51.

9 Heike CL, Hing AV. Craniofacial Microsomia Overview. GeneReviews ${ }^{\mathrm{TM}}$ [Internet]. Seattle, WA: University of Washington, Seattle, 2009.

10 Rooryck C, Souakri N, Cailley D, Bouron J, Goizet C, Delrue MA, Marlin S, Lacombe FD, Arveiler B. Array-CGH analysis of a cohort of 86 patients with oculoauriculovertebral spectrum. Am J Med Genet A 2010;152A:1984-9.

11 Pierpont ME, Moller JH, Gorlin RJ, Edwards JE. Congenital cardiac, pulmonary, and vascular malformations in oculoauriculovertebral dysplasia. Pediatr Cardiol 1982;2:297-302.

12 Cohen MM Jr. Variability versus "incidental findings" in the first and second branchial arch syndrome: unilateral variants with anophthalmia. Birth Defects Orig Artic Ser 1971;7:103-8.

13 Cohen MM Jr, Rollnick BR, Kaye Cl. Oculoauriculovertebral spectrum: an updated critique. Cleft Palate J 1989;26:276-86. 
14 Figueroa $A A$, Pruzansky $S$. The external ear, mandible and other components of hemifacial microsomia. J Maxillofac Surg 1982;10:200-11.

15 Mansour AM, Wang F, Henkind P, Goldberg R, Shprintzen R. Ocular findings in the facioauriculovertebral sequence (Goldenhar-Gorlin syndrome). Am J Ophthalmol 1985:100:555-9.

16 Melnick M. The etiology of external ear malformations and its relation to abnormalities of the middle ear, inner ear, and other organ systems. Birth Defects Orig Artic Ser 1980;16:303-31.

17 Rollnick BR. Oculoauriculovertebral anomaly: variability and causal heterogeneity. Am J Med Genet 1988;(Suppl 4):41-53.

18 Rollnick BR, Kaye Cl, Nagatoshi K, Hauck W, Martin AO. Oculoauriculovertebral dysplasia and variants: phenotypic characteristics of 294 patients. Am J Med Genet 1987;26:361-75.

19 Rollnick BR, Kaye Cl. Hemifacial microsomia and variants: pedigree data. Am J Med Genet 1983;15:233-53.

20 Rollnick BR, Kaye Cl. Hemifacial microsomia and the branchio-oto-renal syndrome. J Craniofac Genet Dev Biol 1985;(Suppl 1):287-95.

21 Shokeir MH. The Goldenhar syndrome: a natural history. Birth Defects Orig Artic Ser 1977;13(3C):67-83.

22 Engiz O, Balci S, Unsal M, Ozer S, Oguz KK, Aktas D. 31 cases with oculoauriculovertebral dysplasia (Goldenhar syndrome): clinical, neuroradiologic audiologic and cytogenetic findings. Genet Couns 2007;18:277-88

23 Kumar A, Friedman JM, Taylor GP, Patterson MW. Pattern of cardiac malformation in oculoauriculovertebral spectrum. Am J Med Genet 1993;46:423-6.

24 Johnson KA, Fairhurst J, Clarke NM. Oculoauriculovertebral spectrum: new manifestations. Pediatr Radiol 1995;25:446-8.

25 Aleksic S, Budzilovich G, Greco MA, McCarthy J, Reuben R, Margolis S, Epstein F, Feigin I, Pearson J. Intracranial lipomas, hydrocephalus and other CNS anomalies in oculoauriculo-vertebral dysplasia (Goldenhar-Gorlin syndrome). C hilds Brain 1984:11:285-97.

26 Szabo-Rogers HL, Smithers LE, Yakob W, Liu KJ. New directions in craniofacial morphogenesis. Dev Biol 2010;341:84-94.

27 Chai Y, Maxson RE Jr. Recent advances in craniofacial morphogenesis. Dev Dyn 2006:235:2353-75.

28 Minoux M, Rijli FM. Molecular mechanisms of cranial neural crest cell migration and patterning in craniofacial development. Development 2010;137:2605-21.

29 Kaye Cl, Martin AO, Rollnick BR, Nagatoshi K, Israel J, Hermanoff M, Tropea B, Richtsmeier JT, Morton NE. Oculoauriculovertebral anomaly: segregation analysis. Am J Med Genet 1992:43:913-7.

30 Tasse C, Majewski F, Böhringer S, Fischer S, Lüdecke HJ, Gillessen-Kaesbach G, Wieczorek D. A family with dominant Oculo-auriculo-vertebral spectrum. Am J Med Genet 2007;78:345-9.

31 Tasse C, Majewski F, Böhringer S, Fischer S, Lüdecke HJ, Gillessen-Kaesbach G, Wieczorek D. A family with autosomal dominant oculo-auriculo-vertebral spectrum. Clin Dysmorphol 2007:16:1-7.

32 Vendramini-Pittoli S, Kokitsu-Nakata NM. Oculoauriculovertebral spectrum: report of nine familial cases with evidence of autosomal dominant inheritance and review of the literature. Clin Dysmorphol 2009;18:67-77.

33 Tsai FJ, Tsai $\mathrm{CH}$. Autosomal dominant inherited oculo-auriculo-vertebral spectrum: report of one family. Zhonghua Min Guo Xiao Er Ke Yi Xue Hui Za Zhi 1993;34:27-31.

34 Goodin K, Prucka S, Woolley AL, Kohlhase J, Smith RJ, Grant J, Robin NH. Familial transmission of Oculo-auriculo-vertebral spectrum (Goldenhar syndrome) is not due to mutations in either EYA1 or SALL1. Am J Med Genet A 2009;(3):535-8.

35 Callier P, Faivre L, Thauvin-Robinet C, Marle N, Mosca AL, D'Athis P, Guy J, Masurel-Paulet A, Joly L, Guiraud S, Teyssier JR, Huet F, Mugneret F. Array-CGH in a series of 30 patients with mental retardation, dysmorphic features, and congenita malformations detected an interstitial 1p22.2-p31.1 deletion in a patient with features overlapping the Goldenhar syndrome. Am J Med Genet $A$ 2008;146A:2109-15.

36 Kelberman D, Tyson J, Chandler DC, Mclnerney AM, Slee J, Albert D, Aymat A, Botma M, Calvert M, Goldblatt J, Haan EA, Laing NG, Lim J, Malcolm S, Singer SL, Winter RM, Bitner-Glindzicz M. Hemifacial microsomia: progress in understanding the genetic basis of a complex malformation syndrome. Hum Genet 2001:109:638-45.

37 Huang XS, Li X, Tan C, Xiao L, Jiang HO, Zhang SF, Wang DM, Zhang JX. Genome-wide scanning reveals complex etiology of oculo-auriculo-vertebral spectrum. Tohoku J Exp Med 2010;222:311-18.

38 Huang XS, Xiao L, Li X, Xie Y, Jiang HO, Tan C, Wang L, Zhang JX. Two neighboring microdeletions of $5 q 13.2$ in a child with oculo-auriculo-vertebral spectrum. Eur J Med Genet 2010;53:153-8.

39 Descartes M. Oculo-auriculo-vertebral spectrum with 5p15.33-pter deletion. Clin Dysmorphol 2006;15:153-4.

40 Josifova DJ, Patton MA, Marks K. Oculo-auriculo-vertebral spectrum phenotype caused by an unbalanced t (5;8) (p15.31; p23.1) rearrangement. Clin Dysmorphol 2004;151-153.

41 Ladekarl S. Combination of Goldenhar's syndrome with the Cri-Du-Chat syndrome. Acta Ophthalmol (Copenh) 1968;46:605-10.
42 Ala-Mello S, Siggberg L, Knuutila S, von Koskull H, Taskinen M, Peippo M. Further evidence for a relationship between the $5 p 15$ chromosome region and the oculoauriculovertebral anomaly. Am J Med Genet A 2008;146A:2490-4.

43 Dabir TA, Morrison PJ. Trisomy 10p with clinical features of facio-auriculo-vertebral spectrum: a case report. Clin Dysmorphol 2006;15:25-7.

44 Rooryck C, Stef M, Burgelin I, Simon D, Souakri N, Thambo JB, Chateil JF, Lacombe D, Arveiler B. 2.3 Mb terminal deletion in 12p13.33 associated with Oculo-auriculo-vertebral spectrum and evaluation of WNT5B as a candidate gene. Eur J Med Genet 2009;52:446-9.

45 Abdelmoity AT, Hall JJ, Bittel DC, Yu S. $1.39 \mathrm{Mb}$ inherited interstitial deletion in 12p13.33 associated with developmental delay. Eur J Med Genet 2011;54:198-203

46 Ballesta-Martínez MJ, López-González V, Dulcet LA, Rodríguez-Santiago B, Garcia-Miñaúr S, Guillen-Navarro E. Autosomal dominant Oculo-auriculo-vertebral spectrum and 14q23.1 microduplication. Am J Med Genet A 2013;161:2030-5.

47 Ou Z, Martin DM, Bedoyan JK, Cooper ML, Chinault AC, Stankiewicz P, Cheung SW. Branchiootorenal syndrome and Oculo-auriculo-vertebral spectrum features associated with duplication of SIX1, SIX6, and OTX2 resulting from a complex chromosomal rearrangement. Am J Med Genet Part A 2008;146A:2480-9.

48 Gimelli S, Cuoco C, Ronchetto P, Gimelli G, Tassano E. Interstitial deletion $14 q 31.1 q 31.3$ transmitted from a mother to her daughter, both with features of hemifacial microsomia. J App/ Genet 2013;54:361-5.

49 Brun A, Cailley D, Toutain J, Bouron J, Arveiler B, Lacombe D, Goizet C, Rooryck C. $1.5 \mathrm{Mb}$ microdeletion in $15 \mathrm{q} 24$ in a patient with mild OAVS phenotype. Eur J Med Genet 2012;55:135-9.

50 Verloes A, Seret N, Bernier V, Gonzales M, Herens C, Koulischer L. Branchial arch anomalies in trisomy 18. Ann Genet 1991;34:22-4.

51 Herman GE, Greenberg F, Ledbetter DH. Multiple congenital anomaly/mental retardation (MCA/MR) syndrome with Goldenhar complex due to a terminal del (22q). Am J Med Genet 1988;29:909-15.

52 Xu J, Fan YS, Siu VM. A child with features of Goldenhar syndrome and a nove $1.12 \mathrm{Mb}$ deletion in 22q11.2 by cytogenetics and oligonucleotide array $\mathrm{CGH}$ : is this a candidate region for the syndrome? Am J Med Genet A 2008;146A:1886-9.

53 Digilio MC, McDonald-McGinn DM, Heike C, Catania C, Dallapiccola B, Marino B, Zackai EH. Three patients with oculo-auriculo-vertebral spectrum and microdeletion 22q11.2. Am J Med Genet A 2009;149A:2860-4.

54 Tan TY, Collins A, James PA, McGillivray G, Stark Z, Gordon CT, Leventer RJ, Pope K, Forbes R, Crolla JA, Ganesamoorthy D, Burgess T, Bruno DL, Slater HR, Farlie PG, Amor DJ. Phenotypic variability of distal 22q11.2 copy number abnormalities. Am J Med Genet A 2011;155A:1623-33.

55 Kobrynski L, Chitayat D, Zahed L, McGregor D, Rochon L, Brownstein S, Vekemans M, Albert DL. Trisomy 22 and facioauriculovertebral (Goldenhar) sequence. Am J Med Genet 1993;46:68-71.

56 Quintero-Rivera F, Martinez-Agosto JA. Hemifacial microsomia in cat-eye syndrome: 22q11.1-q11.21 as candidate loci for facial symmetry. Am J Med Genet A 2013;161:1985-91.

57 Torti EE, Braddock SR, Bernreuter K, Batanian JR. Oculo-auriculo-vertebral spectrum, cat eye, and distal 22q11 microdeletion syndromes: A unique double rearrangement. Am J Med Genet A 2013;161:1992-8.

58 Balci S, Engiz O, Yilmaz Z, Baltaci V. Partial trisomy $(11 ; 22)$ syndrome with manifestations of Goldenhar sequence due to maternal balanced $t(11 ; 22)$. Genet Couns 2006:17:281-9.

59 Rao VA, Kaliaperumal S, Subramanyan T, Rao KR, Bhargavan R. Goldenhar's sequence with associated juvenile glaucoma in Turner's syndrome. Indian J Ophthalmol 2005;53:267-8.

60 Garavelli L, Virdis R, Donadio A, Sigorini M, Banchini G, Balestrazzi P, Fryns JP. Oculo-auriculo-vertebral spectrum in Klinefelter syndrome. Genet Couns 1999;10:321-4.

61 Poonawalla $\mathrm{HH}$, Kaye $\mathrm{Cl}$, Rosenthal IM, Pruzansky S. Hemifacial microsomia in a patient with Klinefelter syndrome. Cleft Palate J 1980;17:194-6.

62 Rooryck C, VuPhi Y, Souakri N, Burgelin I, Saura R, Lacombe D, Arveiler B, Taine L. Characterization of a de novo balanced translocation $t(9 ; 18)(p 23 ; q 12.2)$ in a patient with oculoauriculovertebral spectrum. Eur J Med Genet 2010;53:104-7.

63 Stanojević M, Stipoljev F, Koprcina B, Kurjak A. Oculo-auriculo-vertebral (Goldenhar) spectrum associated with pericentric inversion 9: coincidental findings or etiologic factor? J Craniofac Genet Dev Biol 2000;20:150-4.

64 Northup JK, Matalon D, Hawkins JC, Matalon R, Velagaleti GV. Pericentric inversion, inv(14)(p11.2q22.3), in a 9-month old with features of Goldenhar syndrome. Clin Dysmorphol 2010;19:185-9.

65 Hodes ME, Gleiser S, DeRosa GP, Yune HY, Girod DA, Weaver DD, Palmer CG. Trisomy 7 mosaicism and manifestations of Goldenhar syndrome with unilateral radial hypoplasia. J Craniofac Genet Dev Biol 1981:1:49-55.

66 de Ravel TJ, Legius E, Brems H, Van Hoestenberghe R, Gillis PH, Fryns JP. Hemifacial microsomia in two patients further supporting chromosomal mosaicism as a causative factor. Clin Dysmorphol 2001;10:263-7.

67 Wilson GN, Barr M Jr. Trisomy 9 mosaicism: another etiology for the manifestations of Goldenhar syndrome. J Craniofac Genet Dev Biol 1983;3:313-16. 
68 Robinson LK, Hoyme HE, Edwards DK, Jones KL. Vascular pathogenesis of unilateral craniofacial defects. J Pediatr 1987;111:236-9.

69 Soltan HC, Holmes LB. Familial occurrence of malformations possibly attributable to vascular abnormalities. J Pediatr 1986;108:112-14.

70 Van Allen MI. Structural anomalies resulting from vascular disruption. Pediatr Clin North Am 1992;39:255-77.

71 Werler MM, Sheehan JE, Hayes C, Mitchell AA, Mulliken JB. Vasoactive exposures, vascular events, and hemifacial microsomia. Birth Defects Research (Part A) 2004;70:389-95.

72 Hartsfield JK. Review of the etiologic heterogeneity of the oculo-auriculo-vertebral spectrum (Hemifacial Microsomia). Orthod Craniofac Res 2007;10:121-8.

73 Ewart-Toland A, Yankowitz J, Winder A, Imagire R, Cox VA, Aylsworth AS, Golabi M. Oculoauriculovertebral abnormalities in children of diabetic mothers. Am J Med Genet 2000:90:303-9.

74 Wieczorek D, Ludwig M, Boehringer S, Jongbloet PH, Gillessen-Kaesbach G, Horsthemke B. Reproduction abnormalities and twin pregnancies in parents of sporadic patients with oculo-auriculo-vertebral spectrum/Goldenhar syndrome. Hum Genet 2007;121:369-76.

75 Balci S, Engiz O, Alikasifoglu M, Esinler I, Sinan Beksac M. Association of assisted reproductive technology with twinning and congenital anomalies. Indian J Pediatr 2008;75:638-40.

76 Roesch C, Steinbicker V, Korb C, von Rohden L, Schmitt J. Goldenhar anomaly in one triplet derived from intracytoplasmic sperm injection (ICSI). Am J Med Genet 2001;101:82-38.

77 Cordier J, Stricker M, Reny A, Raspiller A. Discordant Franceschetti-Goldenhar syndrome in 2 monozygotic twins. Arch Ophtalmol Rev Gen Ophtalmol 1970:30:321-8.

78 Gómez García A, Vargas Torcal F, Payá Abad EA. Goldenhar syndrome. Discordance in monozygotic twins. An Esp Pediatr 1984;20:400-2.

79 Lawson K, Waterhouse N, Gault DT, Calvert ML, Botma M, Ng R. Is hemifacial microsomia linked to multiple maternities? Br J Plast Surg 2002;55:474-8.

80 Boles DJ, Bodurtha J, Nance WE. Goldenhar complex in discordant monozygotic twins: a case report and review of the literature. Am J Med Genet 1987;28:103-9.

81 Bock RH. Case of epibulbar dermolipoma with unilateral facial malformation in an uniovular twin. Ophthalmologica 1951;122:86-90.

82 Burck U. Genetic aspects of hemifacial microsomia. Hum Genet 1983:64:291-6.

83 Ebbesen F, Petersen W. Goldenhar's syndrome: discordance in monozygotic twins and unusual anomalies. Acta Paediatr Scand 1982;71:685-7.

84 Haberland M, Mokalled MH, Montgomery RL, Olson EN. Epigenetic control of skull morphogenesis by histone deacetylase 8. Genes Dev 2009;23:1625-30.

85 Fischer S, Lüdecke HJ, Wieczorek D, Böhringer S, Gillessen-Kaesbach G, Horsthemke B. Histone acetylation dependent allelic expression imbalance of BAPX1 in patients with the oculo-auriculo-vertebral spectrum. Hum Mol Genet 2006;15:581-7.

86 Katsanis SH, Jabs EW. Treacher Collins Syndrome. GeneReviews ${ }^{\mathrm{TM}}$ [Internet]. Seattle, WA: University of Washington, 2012.

87 Luquetti DV, Heike CL, Hing AV, Cunningham ML, Cox TC. Microtia: Epidemiology \& Genetics. Am J Med Genet A 2012;158A:124-39.
88 Parry DA, Logan CV, Stegmann AP, Abdelhamed ZA, Calder A, Khan S, Bonthron DT, Clowes V, Sheridan E, Ghali N, Chudley AE, Dobbie A, Stumpel CT, Johnson CA. SAMS, a syndrome of short stature, auditory-canal atresia, mandibular hypoplasia, and skeletal abnormalities is a unique neurocristopathy caused by mutations in Goosecoid. Am J Hum Genet 2013:93:1135-42.

89 Kohlhase J. Townes-Brocks Syndrome. GeneReviews ${ }^{\mathrm{TM}}$ [Internet]. Seattle, WA: University of Washington, 2007.

90 Lalani SR, Hefner MA, Belmont JW, Davenport SLH. CHARGE Syndrome. GeneReviews ${ }^{\mathrm{TM}}$ [Internet]. Seattle, WA: University of Washington, 2006.

91 Smith RJH. Branchiootorenal Spectrum Disorders. GeneReviews ${ }^{\mathrm{TM}}$ [Internet]. Seattle, WA: University of Washington, 2013

92 Gordon CT, Petit F, Oufadem M, Decaestecker C, Jourdain AS, Andrieux J, Malan V, Alessandri JL, Baujat G, Baumann C, Boute-Benejean O, Caumes R, Delobel B, Dieterich K, Gaillard D, Gonzales M, Lacombe D, Escande F, Manouvrier-Hanu S, Marlin S, Mathieu-Dramard M, Mehta SG, Simonic I, Munnich A, Vekemans M, Porchet N, de Pontual L, Sarnacki S, Attie-Bitach T, Lyonnet S, Holder-Espinasse M, Amiel J. EFTUD2 haploinsufficiency leads to syndromic oesophageal atresia. J Med Genet 2012:49:737-46.

93 Voigt C, Mégarbané A, Neveling K, Czeschik JC, Albrecht B, Callewaert B, von Deimling $F$, Hehr A, Falkenberg Smel $M$, König $R$, Kuechler $A$, Marcelis $C$ Puiu M, Reardon W, Riise Stensland HM, Schweiger B, Steehouwer M, Teller C, Martin M, Rahmann S, Hehr U, Brunner HG, Lüdecke HJ, Wieczorek D. Oto -facial syndrome and esophageal atresia, intellectual disability and zygomatic anomaliesexpanding the phenotypes associated with EFTUD2 mutations. Orphanet J Rare Dis 2013;8:110.

94 McDermid HE, Morrow BE. Genomic disorders on 22q11. Am J Hum Genet 2002;70:1077-88.

95 Rosias PR, Sijstermans JM, Theunissen PM, Pulles-Heintzberger CF, De Die-Smulders CE, Engelen JJ, Van Der Meer SB. Phenotypic variability of the cat eye syndrome. Case report and review of the literature. Genet Couns 2001;12:273-82

96 Edelmann L, Pandita RK, Spiteri E, Funke B, Goldberg R, Palanisamy N, Chaganti RS, Magenis E, Shprintzen RJ, Morrow BE. A common molecular basis for rearrangement disorders on chromosome 22q11. Hum Mol Genet 1999:8:1157-67.

97 Butts SC. The facial phenotype of the velo-cardio-facial syndrome. Int J Pediatr Otorhinolaryngol 2009;73:343-50.

98 Ryan AK, Goodship JA, Wilson DI, Philip N, Levy A, Seidel H, Schuffenhauer S, Oechsler H, Belohradsky B, Prieur M, Aurias A, Raymond FL, Clayton-Smith J, Hatchwell E, McKeown C, Beemer FA, Dallapiccola B, Novelli G, Hurst JA, Ignatius J, Green AJ, Winter RM, Brueton L, Brøndum-Nielsen K, Scambler PJ. Spectrum of clinical features associated with interstitial chromosome 22q11 deletions: a European collaborative study. J Med Genet 1997;34:798-804.

99 Verhagen JM, Diderich KE, Oudesluiis G, Mancini GM, Eggink AJ, Verkleij-Hagoort AC, Groenenberg IA, Willems PJ, du Plessis FA, de Man SA, Srebniak MI, van Opstal D, Hulsman LO, van Zutven LJ, Wessels MW. Phenotypic variability of atypical 22q11.2 deletions not including TBX1. Am J Med Genet $A$ 2012:158A:2412-20 


\section{Oculo-auriculo-vertebral spectrum: a review of the literature and genetic update}

Ana Beleza-Meireles, Jill Clayton-Smith, Jorge M Saraiva, et al.

J Med Genet published online August 12, 2014

doi: 10.1136/jmedgenet-2014-102476

Updated information and services can be found at:

http://jmg.bmj.com/content/early/2014/08/12/jmedgenet-2014-102476.full.html

\section{These include:}

References This article cites 89 articles, 7 of which can be accessed free at: http://jmg.bmj.com/content/early/2014/08/12/jmedgenet-2014-102476.full.html\#ref-list-1

$\mathbf{P}<\mathbf{P} \quad$ Published online August 12, 2014 in advance of the print journal.

Email alerting
service

Receive free email alerts when new articles cite this article. Sign up in the box at the top right corner of the online article.

Notes

Advance online articles have been peer reviewed, accepted for publication, edited and typeset, but have not not yet appeared in the paper journal. Advance online articles are citable and establish publication priority; they are indexed by PubMed from initial publication. Citations to Advance online articles must include the digital object identifier (DOIs) and date of initial publication.

To request permissions go to:

http://group.bmj.com/group/rights-licensing/permissions

To order reprints go to:

http://journals.bmj.com/cgi/reprintform

To subscribe to BMJ go to:

http://group.bmj.com/subscribe/ 